\title{
Taxonomy of the genus Commelina Plum. ex L. (Commelinaceae) in Nepal
}

\author{
Jyoti P. Gajurel" and Krishna K. Shrestha
}

Central Department of Botany, Tribhuvan University, Kirtipur, Kathmandu, Nepal; *Corresponding author, email: gajurelomjyoti@yahoo.com

\begin{abstract}
About 170 species of Commelina are known from tropical and subtropical regions of the world. Nepal contributes 6 species of Commelina to the world flora. In the present work, taxonomy of all the six species of Commelina reported from Nepal ( $C$. benghalensis, C. caroliniana, C. diffusa, C. maculata, C. paludosa and C. suffruticosa) was studied. Voucher specimens were collected from Central and Eastern Nepal, covering 14 districts. Morphological characters were studied from these collections. Palynological and anatomical characters were also used to see if they are taxonomically important to delimit the taxa within Commelina. Morphological characters seemed promising to delimit the taxa within Commelina. The key identifying characters at species level are modification in root, form of spathe, structure of leaves and seeds, shape of stomata and pollen. Palynological and anatomical characters were also useful, to some extent, in separating some species, but were not significant as compared to morphological data. Some specimens, close to $C$. benghalensis and $C$. caroliniana, showed very different characters. Thus further study is needed to confirm their taxonomic status.
\end{abstract}

Key-words: anatomy, flora, morphological characters, palynology, voucher specimens.

\section{Introduction}

Commelinaceae, commonly known as the spiderwort family, is a medium size family with 41 genera and 650 species worldwide (Kubitzia 1998). The members of the family Commelinaceae are distributed worldwide, mostly in tropics and subtropics with considerable diversity extending into northern temperate regions. In Nepal, 9 genera and 22 species have been enumerated within Commelinaceae (Press et al. 2000).

The major centers of diversity for the family Commelinaceae are Mexico (especially Oaxaca and Chiapas) and north Central America (especially for the subtribe Tradescantiinae and Thysantheminae); tropical Africa (including Madagascar); and peninsular Thailand and southwestern China (especially for Commelinae, Cynotinae and Streptoliriinae). The members of the family Commelinaceae are ecologically diverse (Kubitzia 1998). They are found chiefly in humid and mesic habitats, such as forests and grasslands. The greatest diversity has been recorded in Africa, where, along with Madascar, nearly half of the genera and about $40 \%$ of the species are found (Faden 1983). There is natural division of the taxa between Old and the New World (Faden 1985).

Commelina communis, commonly known as the Asiatic dayflower, serves as the type for the family Commelinaceae. It is native to East Asia and extending into South East Asia. It has also been introduced to parts of Europe and much of North America. Linnaeus picked the name Commelina in honor of the three Dutch brothers of the Commelijn family, using the flower of Commelina communis to symbolize them. The large, blue petals represent the botanists Jan Commelijn and his nephew Kaspar, who were famous for their work, while the small lower white petal symbolizes their brother who died at a young age (http://en.wikipedia.org/wiki/ Commelina_caroliniana).

Older classification of Commelinaceae relied heavily on floral features. However, Faden and Hunt (1991) proposed a new classification above generic level based on anatomical, palynological and cytological characters, in addition to morphological characters. Within Commelinaceae, two sub-families (Cartonematoideae and Commeliinoideae) were recognized. The sub-family Commelinoideae has been divided into two tribes, Tradescantieae and Commelineae. Cladistic studies by Evans and Faden (2000) revealed inadequecy of morphological characters indemonstrating relationships among the genera. Instead, DNA sequence data, using the rbcL gene has been found to be much more helpful (Evans and Faden 2000).

About 170 species of Commelina are known from tropical and subtropical regions of the world. Nepal contributes 6 species of Commelina to the world flora. In the present work, taxonomy of all the six species of Commelina reported from Nepal (C. benghalensis, C. caroliniana, C. diffusa, C. maculata, C. paludosa and C. suffruticosa) was studied mainly based on morphological characters. In addition, palynological and anatomical characters were also used to see if they are taxonomically important to delimit the taxa within Commelina.

\section{Materials and Methods}

\section{COLLECTION AND PRESERVATION}

The specimens of Commelina were collected from different locations in central and eastern Nepal, covering 14 districts. Plant 
specimens with their reproductive parts were collected as far as possible and photographs were taken. Field notes of all collections were recorded in the field. As the flowers were very difficult to preserve, a blotter was kept below the flowers and covered by nonsticky transparent plastic. Colors of petals retained even for more than a year by preserving the flower through this process.

The plant parts collected for anatomical study were liquidpreserved [ 1 part by volume formalin solution: 1 part glacial acetic acid: 18 parts $70 \%$ ethanol].

\section{MORPHOLOGICAL STUDY AND IDENTIFICATION}

The taxonomic treatment of the genera and species were based on voucher specimens collected by the authors as well as those housed at Tribhuvan University Central Herbarium (TUCH) and National Herbarium and Plant Laboratories (KATH). The morphological study of the herbarium specimen was done with the help of stereomicroscope and dissecting-microscope. The herbarium specimens were identified with the help of available literature. Some specimens were verified as well as identified by Dr. Robert B. Faden, Department of Botany, Smithsonian Institution, and Washington DC, USA. During identification, the collected specimens were compared with specimens deposited at TUCH and KATH.

\section{ANATOMICAL AND PALYNOLOGICAL STUDY}

Anatomical study of stem and leaf was carried out by following the procedure mentioned in Faden and Inman (1996). Since the chemicals required for analysis of pollen by acetolysis and enzymebased methods are unavailable, common chemical benzyol peroxide was used to study the pollen in some species of Commelina. The slides were prepared by standard procedure (Hunt and Poole 1979).

\section{Results}

\section{MORPHOLOGICAL VARIATION}

The members of the genus Commelina are usually terrestrial and herbaceous. Majority of them are perennial while some are annual. The roots are mostly fibrous. However, roots of Commelina maculata and Commelina suffruticosa are tuberous. The stem is elongated, rounded, solid, nodes swollen or brittle in almost all genera and species of Commelina. The leaves are simple, cauline, spiral, petiolate, sheathing at base, and sheaths (the summit of the sheath of Commelina benghalensis, C. maculata and C. paludosa bear reddish brown hairs) are closed. The spathe of Commelina benghalensis, C. maculata and C. paludosa is funnel shaped (margin fused), while that of $C$. diffusa, C. caroliniana and $C$. suffruticosa is conduplicate. Similarly, the spathe of $C$. benghalensis, C. maculata and $C$. suffruticosa is pubescent, while that of C. paludosa, C. diffusa and C. caroliniana is glabrous. The flowers are usually blue; however, variation also occurs in some species from deep blue to faint blue. The fruit of Commelina is a capsule and it may be of various shapes ranging from oblong, elliptic to obovoid. The seeds are cylindric or ellipsoid, reticulate or sub-smooth, and hilum is linear.

Anatomical study of stem revealed 3-celled hook like epidermal hairs with pointed end. The hypodermis was few layered sclerenchymatous. The vascular bundles were conjoint, collateral, endarch and closed, large in size and were distributed away from the pith. The metaxylems showed larger cavity. The stomata were 6 celled, brachyparahexacytic. The shape of the stomata due to arrangement of 6 cells was either circular (C. benghalensis, $C$. maculata and C. suffruticosa) or rectangular (C. caroliniana, $C$. diffusa and C. paludosa).

Regarding palynological study, in Commelina the tectum was perforate, irregularly spaced. Spinulae were closer towards transitional zone. Sulcus was spinolate. Kidney-shaped pollen was found in C. benghalensis; whereas in C. paludosa, the shape was circular and in C. caroliniana, C. diffusa and C. maculata the shape was oval.

\section{TAXONOMIC TREATMENT}

\section{COMMELINACEAE R. Br., Prod. 268 (1810).}

Type: Commelina Plum. ex L., Gen. Pl. ed. 5.25 (1754).

Herbs annual or perennial. Leaves alternate, sheaths closed, nodes swollen. Inflorescence panicle or coiled cyme or rarely solitary. Flowers bisexual, actinomorphic or zygomorphic, bracts modified as spathe. Sepals 3, membranous. Petals 3, longer, one clawed, and blue (faint, deep) or white or pink. Stamens 3-6, basifixed, filaments glabrous or pubescent, staminodes 0-3. Carpels 2-3 loculed, ovary superior, style long, axile placentation. Capsules 23 valved. Seeds few, large, hilium linear or orbicular.

Commelina Plum. ex. L., Gen. Pl. ed. 5.25 (1754).

Type: Commelina communis L., Sp. Pl. 1: 40-41 (1753).

Herbs annual or perennial. Roots thin or tuberous. Rhizomes absent. Stem slender and creeping below. Leaves spiral to alternate, base oblique, lanceolate, acuminate. Inflorescence terminal, cymes enclosed in spathe, upper cyme single flowered, lower cymes several flowered. Flowers bisexual and staminate, zygomorphic. Sepals 3, membranous. Petals 3, longer, one clawed, lower petal is faintly blue. Stamens 3-6, basifixed, filaments glabrous or pubescent, staminodes 0-3. Carpels 2-3 loculed ovary, style long, axile placentation. Capsules bi-tri locular, 1-2 seeded. Seeds cylindric or ellipsoid, reticulate or sub-smooth, hilum linear.

\section{Key to the species of Commelina}

1.a. Spathe funnel form, cincinni larger ................................. 2

1.b. Spathe conduplicate, cincinni smaller....................................... 4

2.a. Spathe pubescent, petals smaller ….......................................... 3

2.b. Spathe glabrous, petals larger ............................. C. paludosa

3.a. Roots fibrous, cleistogamous flowers present ......... benghalensis 
3.b. Roots tuberous, cleistogamous flowers absent............. C. maculata 4.a. Spathe single, tuberous roots absent .. .5 4.b. Spathe in cluster, tuberous roots present ............... C. suffruticosa 5.a. Capsule 3 valved, antherode with central dark spot on surface, seeds reticulate C. diffusa

5.b. Capsule 2 valved, antherode with smooth surface, seeds smooth ..C. caroliniana

Commelina benghalensis L., Sp. Pl. 1: 41 (1753). Hook, Fl. Brit. Ind. 6: 370 (1892). Hara, Enum. Fl. Pl. Nep. 1: 82 (1978). Noltie, Fl. Bhutan 3 (1): 223 (2000). Press et al., Ann. Check. Fl. Nep.: 48 (2000). Deyuan and DeFilipps, Fl. China 24: 67 (2000).

Type: Commelina cucullata L., Mant 176 (1771). Herbs perennial, 20-36 cm. Stems slender and creeping below. Leaves spirally arranged, 3-6 × 1.5-4 cm, base oblique, ovate, acute, sheath with reddish-brown hairs. Inflorescence terminal, 1 or cymes enclosed in funnel shaped spathe, upper cyme staminate, lower cymes several flowered. Flowers perfect and staminate, zygomorphic, cleistogamous flowers at base. Sepals 3, 3-4 mm, membranous. Petals 3, 0.4-1.2 cm, longer, one clawed, lower petal faintly blue. Stamens 6, 4-6 mm, basifixed, filaments pubescent, staminodes 3. Carpels 2-3 loculed ovary, 4-6 mm, superior, style long. Capsules oblong-elliptic, 3-valved. Seeds black, cylindric or semicylindric, rugose, irregularly reticulate. Fl. Jun.-Aug. \& Fr. Aug.Sept. (Fig. 1).

Ecology: Common on moist and shady places.

Distribution: Nepal (WCE), 100-1800 m, Himalaya, East India to China, Japan, Malaysia, Africa.

Note: This species can be easily identified by the presence of cleistogamous flower near the ground. Plants collected from different localities showed variation in leaf size and shape. Some collections had smaller sized leaves, whereas some had relatively larger sized leaves. Some collections showed sky blue (slightly light) petals and some collections had deep blue petals. The summit of the sheath in some collections had reddish brown hairs. The flowering time was usually 6 am to $12 \mathrm{pm}$ and flowers faded out when exposed to sunlight.

Shape and arrangement of leaves of some specimens closely resembled with those of $C$. benghalensis. However, these specimens differed from the latter species in having comparatively longer sheath, diffused stem, and 3-seeded capsule. These characters, however, were close to $C$. ensifolia $\mathrm{R}$. Br. Thus, these specimens need through study to determine their taxonomic status.

Specimens examined: Western Nepal: Kailali, Seti, $900 \mathrm{~m}$, 1978.09.31, Raunior et al. 1904 (TUCH, KATH). Jumla, near bazaar, 800 m, 1982.09.12, N.P. Manandhar 9037 (TUCH, KATH). Central Nepal: Manang, Chame, 1600 m, 1983.08.12, N.P. Manandhar 9806 (KATH). Kathmandu, on the way to Gokarna temple, 1400 m, 2006.09.01, J.P. Gajurel 177 (TUCH, KATH). Kirtipur, 1320 m, 2006.08.21, J.P. Gajurel 146 (TUCH, KATH). Airport to Tilganga, 1400 m, 2006.08.20, J.P. Gajurel 139 (KATH, TUCH). Chovar, 1550 m, 2006.08.14, J.P. Gajurel 118 (KATH, TUCH). Dakshinkali entry gate to temple, $1650 \mathrm{~m}, 2006.08 .29$ J.P. Gajurel 172 (KATH, TUCH). Sundarijal buspark to Barak, 1450 m, 2006.08.04, J.P.
Gajurel et al., 98 (KATH, TUCH). Budhanilkhanta to Shivapuri, 1900 m, 2006.09.01, J.P. Gajurel, 182 (KATH, TUCH). Lalitpur, Godawari to temple area, 1550 m, 2006.08.22, J.P. Gajurel 147 (TUCH, KATH). Lamatar-6 to Sisnary, 1600 m, 2006.09.07, J.P. Gajurel 200 (TUCH, KATH). Bhaktapur, Kamal Vinayak, 1400 m, 2006.08.01, J.P. Gajurel, 90 (KATH, TUCH). Changunarayan, 1600 m, 2006.10.13, J.P. Gajurel 257 (TUCH, KATH). Kavre, Dolalghat buspark to picnic spot, 700 m, 2006.09.03, J.P. Gajurel 187 (TUCH, KATH). Phulbari-5, 1500 m, 2006.08.09, J.P. Gajurel et al., 107 (KATH, TUCH). Phulbari-5, 1500m, 2006.08.10, J.P. Gajurel et al.107 (TUCH, KATH). Eastern Nepal: Sunsari, Titragachi, 100 m, 1993.05.31, P.R. Shakya et al. 289 (KATH).

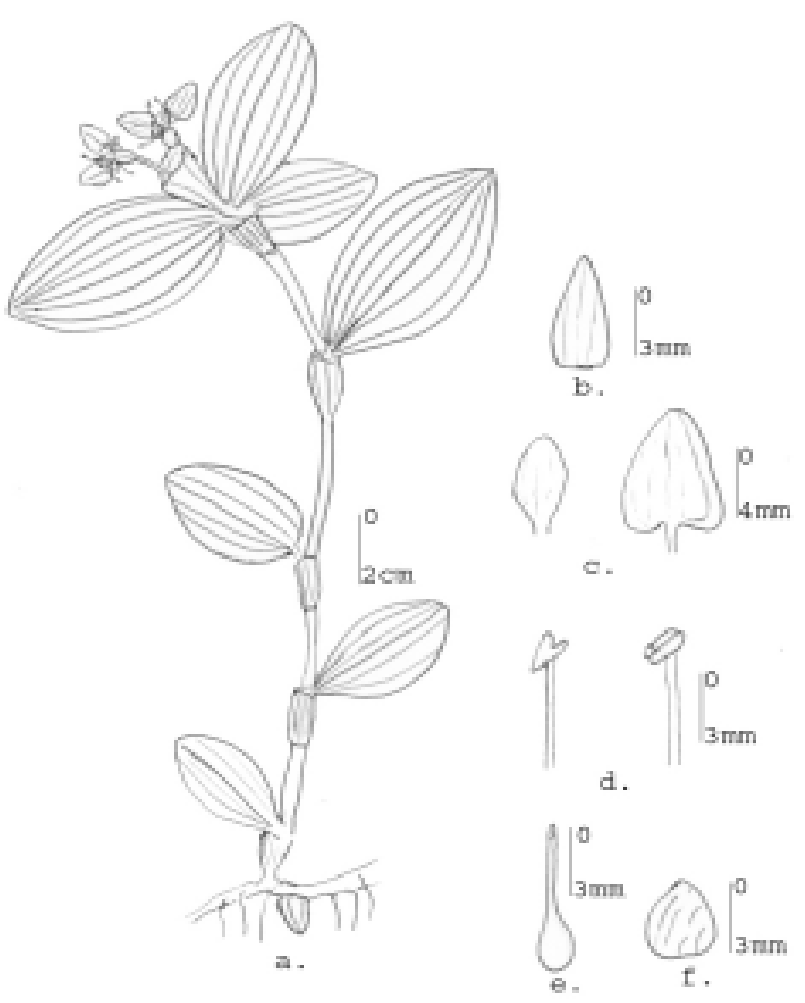

Fig.1. Commelina benghalensis L.: (a) habit sketch, (b) sepal, (c) petal, (d) stamens, (e) carpel, (f) seed (J.P. Gajurel 118).

Commelina caroliniana Walter, Flora Caroliniana, Secundum: 68 (1788). Faden, Taxon. 38 (1): 43-53 (1989). Noltie, Fl. Bhutan 3 (1): 237 (2000).

Commelina hasskarlii C. B. Clarke, Commel. Cyrt. Bengal.: 13, t. 3 (1874). Hara, Enum. Fl. Pl. Nep. 1: 81 (1978).

Herbs annual, 20-70 cm. Stems diffusely branched, decumbent. Leaves alternate, lanceolate, $3.5-7 \times 1-1.4 \mathrm{~cm}$, acute to acuminate, glabrous. Inflorescences distal cyme, solitary, spathe conduplicate. Flowers bisexual. Sepals 3, 2-4 mm, free. Petals 3, 0.4-1 cm, free, two blue, one transparent. Stamens 6, 5-7 mm, with white connective, staminodes, antherodes yellow, central spot, cruciform. Carpels 3 loculed ovary, 4-7 mm, style long. Capsules 3-locular, 2-valved. Seeds 1 per valve, dark brown, smooth to faintly alveolate. Fl. Jun.Aug. \& Fr. Aug.-Sept. (Fig. 2).

Ecology: In wet field. 
Distribution: Nepal (CE), 100-1600 m, Himalaya (Kumaun to Assam), India.

Notes: The antherodes have central dark spot. The spot was purple when seen in the fresh plant. In herbarium, the central spot was dark. The plant almost looks like $C$. diffusa, but the character of antherodes and seeds separate this species from $C$. diffusa. This species was dominant in places with high organic matter.

Some specimens had lanceolate leaves, appendiculata and conduplicate spathe, capsule 2 locular with one seed each and seeds with appendage at both ends. Some of these characters closely resembled with that of Commelina caroliniana. However, appendiculata and conduplicate spathe along with seeds having appendage at both ends help in establishing relationship of these specimens with $C$. appendiculata. These specimens therefore need further study.

Specimens examined: Central Nepal: Kathmandu, Maithan, Sankhu, 1500 m, 2006.09.14, J.P. Gajurel, 209 (TUCH, KATH); Kathmandu, Kirtipur, Library, 1340 m, 2006.09.12, J.P. Gajurel 206 (TUCH, KATH). Champadevi from Tindevi temple, 1550 m, 2006.08.16, J.P. Gajurel et al. 133 (TUCH, KATH). On the way to Gokarna temple, 1400 m, 2006.09.01, J.P. Gajurel 178 (TUCH, KATH). Kavre, from Phedi to Namobuddha, 1650 m, 2006.10.04, J. P. Gajurel et al. 231(TUCH, KATH). Eastern Nepal: Rauthat, Chandranegahapur, 120 m, 2006.10.30, J.P. Gajurel 297 (TUCH, KATH). Jhapa, Char-Aali to Army Barak, 100 m, 2006.10.26, J.P. Gajurel 280 (TUCH, KATH). Garamuni, 100 m, 2006.10.26, J.P. Gajurel 276 (TUCH, KATH).

Commelina diffusa N.L. Burman, Fl. Indica. 18, pl. 7, fig. 2 (1768). Hara, Enum. Fl. Pl. Nep. 1: 82 (1978). Press et al., Ann. Check. Fl. Nep.: 48 (2000). Deyuan and DeFilipps, Fl. China 24: 36 (2000).

Commelina communis Dalz \& Gibs., Bombay Fl. 252 (1861).

Commelina nudiflora Hook. f., Fl. Brit. India 6: 369 (1892).

Commelina salciflora Thw., Enum. Pl. Zeyl. 321 (1864). Faden, Rev. Handb. Fl. Cey. 4: 182 (2000).

Herbs annual, 30-40 cm. Stems creeping, branched, glabrous. Leaves sub sessile, leaf sheath hispid, 3-7.5 × 1-1.5 cm, linearlanceolate, glabrous, rounded, acuminate. Inflorescence terminal, cyme, cincinni branched, pedicels thick, curved, involucre bract lanceolate spathe conduplicate. Flowers bisexual. Sepals 3, 3-4 mm, free, membranous. Petals 3, 4-7 mm, blue, conclorous. Stamens 6, 4$6 \mathrm{~mm}$, basifixed, staminodes 3. Carpels 3 loculed ovary, 4-6 mm, superior, style long. Capsules oblong, trigonous, 3-valved. Seeds 1 per valve, black, ovoid-globose, reticulate. Fl. May-Nov. \& Fr. Oct.-Sep. (Fig. 3).

Ecology: Diffusely spreading in margins of rice fields and wet places.

Distribution: Nepal (CE), 100-1600 m, Pantropic and warmtemperate regions.

Note: This species closely resembled with $C$. caroliniana based on leaf morphology and shape of spathe. However, in $C$. diffusa, the anthers were entirely yellow and there was no dark spot in the anther. The seeds were reticulate in C. diffusa which is the diagnostic character separating it from C. caroliniana.

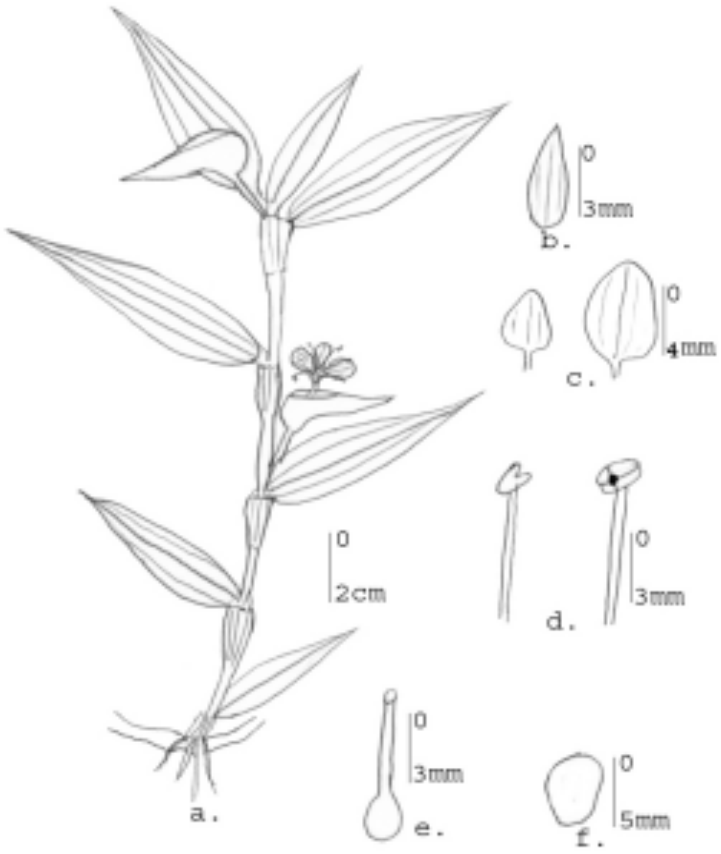

Fig.2. Commelina caroliniana Walter: (a) habit sketch, (b) sepal, (c) petal, (d) stamens, (e) carpel, (f) seed (J.P. Gajurel 206).

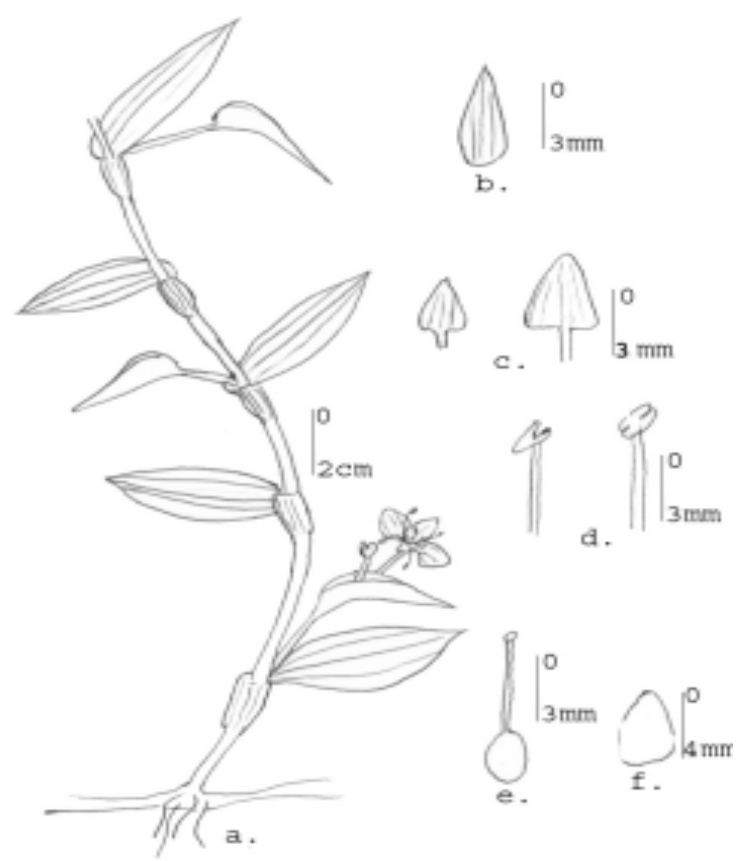

Fig.3. Commelina diffusa N. L. Burman: (a) habit sketch, (b) sepal, (c) petal, (d) stamens, (e) carpel, (f) seed (J.P. Gajurel 286).

Specimens examined: Central Nepal: Lalitpur, Tikabhairab to Chapagaoun, 1550 m, 2006.10.07, J.P. Gajurel et al. 238 (TUCH, KATH). Kavre, Panauti riverside, 1500 m, 2006.08.26, J.P. Gajurel 161 (TUCH, KATH). Chitwan, Ratnanagar Tikauli Jungle, 150 m, 2006.10.09, J.P. Gajurel 253 (TUCH, KATH). Sauraha, 150 m, 2006.10.09, J.P. Gajurel 250 (TUCH, KATH). Eastern Nepal: Udayapur, Beltar, 160 m, 1995.10.21, M. Mikage et al. 9552990 (KATH). Saptari, Koshi Tappu, 60 m, 1995.10.21, M. Mikage et al. 9552911 (KATH). Rauthat, Chandranegahapur, $120 \mathrm{~m}$, 2006.10.30 , J.P. Gajurel 296 (TUCH,KATH). Dharan-2, near bus park, 400 m, 2006.10.29, J.P. Gajurel 94 (TUCH, KATH). Ilam, Ilam bazaar, 1250 m, 2006.10.27, J.P. Gajurel 286 (TUCH, KATH). 
Commelina maculata Edgew., Trans. Linn. Soc. Lond. 20: 89 (1846). Hara, Enum. Fl. Pl. Nep. 1: 82 (1978). Noltie, Fl. Bhutan 3 (1): 225 (2000). Press et al., Ann. Check. Fl. Nep.: 48 (2000). Deyuan and DeFilipps, Fl. China 24: 37 (2000).

Commelina obliqua var. viscida C.B. Clarke, Commel. Cyrt. Bengal: 19, t. 10 (1874).

Commelina paludosa var. viscida (C.B. Clarke) Rao \& Kammathy, Bull. Bot. Surv. India 3: 168 (1961).

Herbs perennial, 9-29 cm. Roots thin to tuberous. Stems creeping proximally, branched, slender, sheath reddish brown. Leaves alternate, 3-7.5 × 1-1.7 cm, sheaths brown ciliate at mouth, ovate-lanceolate or lanceolate, oily surface to glabrous. Inflorescence terminal cyme, cincinni 2-several flowers, spathe pubescent and funnel form, pedicellate. Flowers zygomorphic. Sepals 3, 3-5 mm, membranous. Petals 3, 0.6-1 cm, blue. Stamens 36, 4-6 mm, basifixed, filaments hairy, staminodes 0-3. Carpels 2-3 loculed ovary, 5-6 mm, superior, style long, Capsules globose, trigonous, 3-valved. Seeds 1 per valve, gray-black, ellipsoid, flattened, smooth. Fl. Jun.-Aug. \& Fr. Aug.-Sept. (Fig. 4).

Ecology: Found in moist places.

Distribution: Nepal (CE), 700-1900 m, Himalaya, India, Ceylon, China, Taiwan, Burma, Malaysia.

Note: The leaves in some collections had oily surface (collections from cold places, on way to Tatopani Custom Office). Anthers with dark centre and smooth seeds were seen in some specimens. In some collections, the sheaths had dense brown to reddish brown
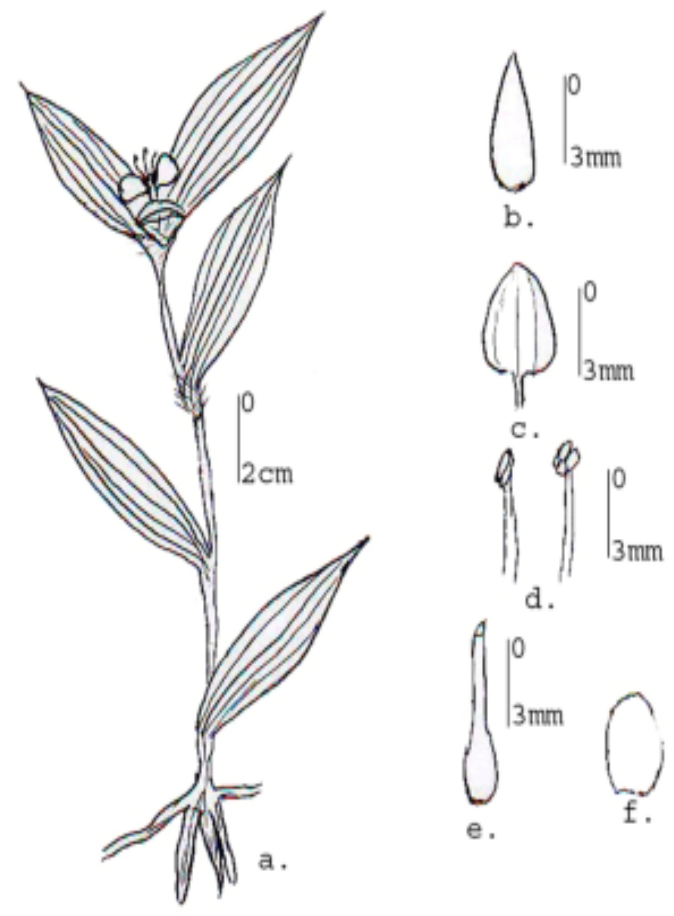

d.
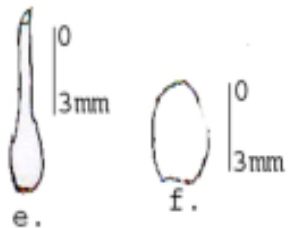

Fig.4. Commelina maculata Edgew.: (a) habit sketch, (b) sepal, (c) petal, (d) stamens, (e) carpel, (f) seed (J.P. Gajurel 156). long hair at the summit, which confused with C. paludosa. There was variation in pubescent on stem. Some were densely pubescent while some were slightly pubescent. However, the hairs were reddish-brown in color.

Specimens Examined: Central Nepal: Nuwakot, Ranipauwa, 1900 m, 2006.09.02, J.P. Gajurel 185 (TUCH, KATH). Kathmandu, on way to Dakshinkali temple, 1600 m, 2006.08.02, J.P. Gajurel 94 (TUCH, KATH). Sundarijal buspark to Barak, 1500 m, 2006.08.04, J.P. Gajurel et al. 102 (TUCH, KATH). Kirtipur Bista Gaon to Tindevi temple, 1550 m, 2006.08.16, J.P. Gajurel et al. 122 (TUCH, KATH). Maithan, Sankhu, 1450 m, 2006.09.14, J.P. Gajurel 209 (TUCH, KATH). Bhudhanilkhanta to Shivapuri, 1900 m, 2006.11.02, J.P. Gajurel et al. 300 (TUCH, KATH). Airport to Tilganga, 1400 m, 2006.08.20, J.P. Gajurel 138 (TUCH, KATH). Chovar, 1550 m, 2006.08.14, J.P. Gajurel 119 (TUCH, KATH). Lalitpur, Godawari to temple area, $1550 \mathrm{~m}, 2006.08 .24$, J.P. Gajurel 153 (TUCH, KATH). Lalitpur, Hatiban, Kirat forest, 1200 m, 2006.09.28, J.P. Gajurel 225 (TUCH, KATH). Tikabhairab to Chapagaoun, 1550 m, 2006.10.07, J. P. Gajurel et al. 240 (TUCH, KATH). Godawari to temple area, 1550 m, 2006.08.24, J.P. Gajurel 152 (TUCH, KATH). Chapagaun buspark, 1550 m, 2006.09.05, J.P. Gajurel 192 (TUCH, KATH). Bhaktapur, Kamal Vinayak, 1400 m, 2006.08.01, J.P. Gajurel 85. Kavre, Panauti riverside, 1500 m, 2006.08.26, J.P. Gajurel 165 (TUCH, KATH). On way to Khopasi powerhouse, 1500 m, 2006.09.27, J.P. Gajurel 221 (TUCH, KATH). Phulbari-5, 1500 m, 2005.07.26, J.P. Gajurel et al. 27 (TUCH, KATH). Dolalghat buspark to picnic spot, 700 m, 2006.09.03, J.P. Gajurel 190 (TUCH, KATH). Sindupalchok, Tatopani to Custom office, 1400 m, 2006.10.20, J.P. Gajurel 270 (TUCH, KATH). Barabise, buspark, 1200 m, 2006.10.21, J.P. Gajurel, 272 (TUCH, KATH). Dolakha, Charikot to Dolakha Bhimsen, 1500 m, 2006.10.15, J. P. Gajurel et al. 259 (TUCH, KATH). Charikot to Kalinchok, 1700 m, 2006.10.16, J. P. Gajurel et al. 263 (TUCH, KATH). Eastern Nepal: Dhankuta, near bus park, Hile, 1900 m, 2006.10.29, J.P. Gajurel 292 (TUCH, KATH).

Commelina paludosa Blume, Enum. Pl. Jav. 1: 2 (1827). Hara, Enum. Fl. Pl. Nep. 1: 82 (1978). Noltie, Fl. Bhutan 3 (1): 235 (2000). Press et al., Ann. Check. Fl. Nep.: 48 (2000). Deyuan and DeFilipps in Fl. China 24: 37 (2000).

Commelina donii A. Dietr., Sp. Pl. 6: 2. 359. 1839.

Commelina obliqua Buch.-Ham. ex D. Don, Prodr. Fl. Nep. 45 (1825).

Herbs perennial, 45-60 cm, robust. Stems erect, branched, glabrous. Leaves alternate, 3.5-13.5 × 1-3 cm, sessile sheath dense reddish brown long hairs, lanceolate. Inflorescence terminal cyme, cincinnus 1 , spathe glabrous and funnel form. Flowers bisexual, 1 to several, pedicellate. Sepals 3, 2-3 mm, membranous, free. Petals 3, 0.3-1.5 cm, free, blue, one light blue. Stamens 6, 4-6 mm, staminodes 0-3. Carpels 3-locular ovary, 4-6 mm. Capsules ovoid-globose, trigonous 3-valved. Seeds 1 per valve, dark brown, ellipsoid, finely reticulate. Fl. Aug.-Sept. \& Fr. Sept.-Oct. (Fig. 5).

Ecology: In moist, shady forest margin.

Distribution: Nepal (WCE), 150-3500 m, Himalaya, India, Ceylon, Indo-China, Taiwan, Burma, Malaysia.

Notes: Plant was robust with largest leaves and petals among taxa of Commelina. Their sheaths had dense reddish brown long hair at the summit but the spathe was glabrous which differentiate 
them from other species of Commelina

Specimens examined: Western Nepal: Bajhang, bazar, $1200 \mathrm{~m}$, 1976.07.28, N.P. Manandhar 26 (KATH). Bardia, near Bardia National Park, 150 m, 1992.11.12, Sajani 19 (KATH). Central Nepal: Kathmandu, Bhudhanilkhanta to Shivapuri, 1900 m, 2006.11.02, J.P. Gajurel et al. 299 (TUCH, KATH). Eastern Nepal: Taplejung, Tamur valley, 1100 m, 1951.08.03, J.D.A. Stainton 1256 (KATH).

Commelina suffruticosa Blume, Enum. Pl. Jav. 1: 3 (1827). Hook, Fl. Brit. Ind. 6: 374 (1892). Hara, Enum. Fl. Pl. Nep. 1: 82 (1978). Noltie, Fl. Bhutan 3 (1): 236 (2000). Press et al. in Ann. Check. Fl. Nep.: 48 (2000). Deyuan and DeFilipps in Fl. China 24: 36 (2000).

Spathodithyros suffruticosus (Blume) Hassk., Comm. Ind. 11. (1870).

Herbs perennial, 20-36 cm. Roots tuberous. Stems erect, branched, glabrous. Leaves alternate, $3.5-13.5 \times 1-3 \mathrm{~cm}$, sheaths ciliate, lanceolate to ovate-lanceolate, glabrous. Inflorescence terminal cyme, cincinni 4-flowered, spathe conduplicate, pedicellate, involucre bract obtuse at apex. Flowers bisexual. Sepals 3, 3-5 mm, membranous. Petals 3, 4-6 mm, purple. Stamens 6, 3 staminodes. Carpels 3-locuar ovary. Capsules globose, 2-valved. Seeds 1 per valve, light brown, ellipsoid, rugose. Fl. Aug.-Sept. \& Fr. Sept.-Oct. (Fig. 6).

Ecology: Dominant inside of forest.

Distribution: Nepal (C), c. 150 m, Himalaya, India, Malaysia.

Notes: The leaves were relatively larger. The spathes were densely clustered and pubescent. Similarly, the tuberous roots were also present.

Specimen examined: Central Nepal: Chitwan, Ratnanagar, Tikauli Jungle, 150 m, 2006.10.09, J.P. Gajurel, 252 (TUCH, KATH).

\section{Discussion}

In most of the taxonomic treatment of Commelina, only morphological traits have been given importance. However, anatomical and palynological attributes could also be significant if considered with morphological traits (Tomlinson 1969). In the species of Commelina, morphological characters like root, leaves, spathe, seeds, etc. seemed useful in separating species. There is also variation in pubescence on the spathe in the $C$. maculata and $C$. benghalensis. The colours of the hairs are also varying among the specimens belonging to $C$. maculata and $C$. benghalensis collected from different places. The colour ranges from reddish brown to slightly brown. These minute morphological characters were given less importance and most of the taxonomic treatments lack them.

Distinguishing Commelina diffusa from C. caroliniana ( $C$. hasskarlii in the older literature) is very difficult. The fruits and flowers can help in separating these two species. The fruits and seeds of $C$. caroliniana are larger than those of $C$. diffusa, and its seeds have a smooth testa, whereas those of $C$. diffusa have reticulate testa. This is very important character found during the

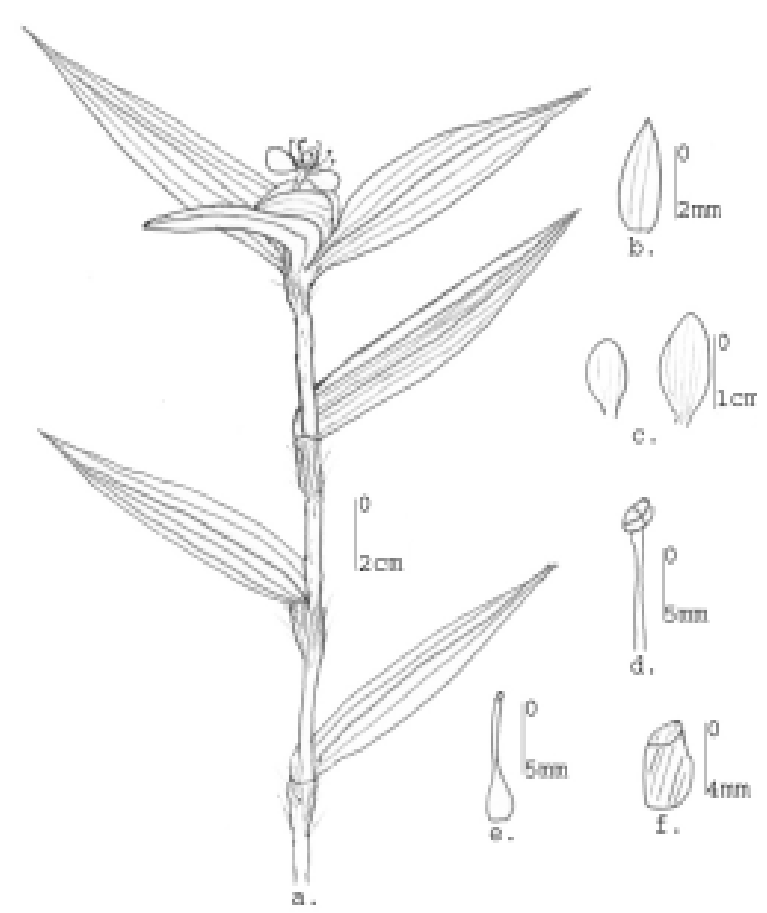

Fig.5. Commelina paludosa Blume: (a) habit sketch, (b) sepal, (c) petal, (d) stamen, (e) carpel, (f) seed (J.P. Gajurel 299).

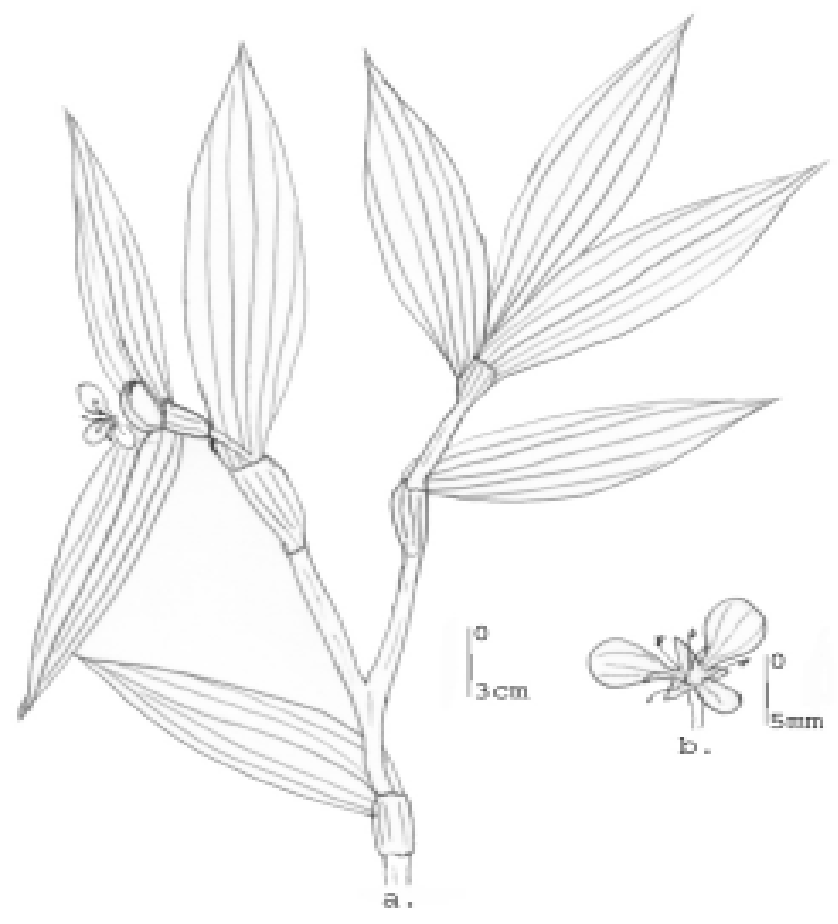

Fig.6. Commelina suffruticosa Blume: (a) habit sketch, (b) flower (J.P. Gajurel 252).

study and can be a useful character to separate the species. These characters do not match with description in Flora of China (Deyuan and DeFilipps 2000) and Flora of Bhutan (Noltie 2000). C. suffruticosa, with tuberous roots, blue flowers and larger leaves with more spathe together, does not agree with the description as in the Flora of China. 
The species of Commelina have been recorded from Western, Central and Eastern regions of Nepal and from tropical to subalpine zones (Press et al. 2000). Commelina diffusa is reported from Central Nepal as well. The range of elevation in which most of the species are found is $1000-1900 \mathrm{~m}$. C. benghalensis and C. maculata are the most common among six species studied.

The stomata are six celled, brachyparahexacytic. This feature matches the findings of Tomlinson (1966). The shape of the stomata due to arrangement of six cells is either circular ( $C$. benghalensis, C. maculata, and C. suffruticosa) or rectangular (C. diffusa, C. paludosa and C. caroliniana). Anatomical finding revels that $C$. benghalensis and C. maculata are closer. Similarly, C. diffusa and C. caroliniana are anatomically closer. Palynology also helped in dividing the species into three major groups on the basis of shape of the pollen (type 1: kidney shape; type 2: circular shape and type 3: oval shape). However, the distinct features were not observed due to lack of Scanning Electron Microscope.

It is confirmed from the study that there are six species of Commelina in Nepal. From the present study, the morphological characters seemed promising to delimit the taxa within Commelina. Palynological and anatomical characters were also useful, to some extent, in separating some species, but were not significant as compared to morphological data. Some specimens, close to $C$. benghalensis and $C$. caroliniana, showed very different characters. Thus further study is needed to confirm their taxonomic status.

\section{Acknowledgements}

We are very much thankful to Prof. Dr. Ram P. Chaudhary (Plant Systematics Unit, Central Department of Botany, Tribhuvan University),
Dr. Keshab Rajbhandary (Former Scientific officer, Department of Plant Resources, Government of Nepal), Dr. Mark Watson (Royal Botanic Garden Edinburgh) and Mr. Bhaskar Adhikary (Royal Botanic Garden, Edinburgh) for their suggestions. Dr. Robert Faden (Department of Botany, Smithsonian Institution) deserves special thanks for helping me with essential literatures and providing useful information on Commelina.

\section{References}

Deyuan H. and DeFilipps R.A. 2000. Flora of China. Science Press China, Beijing and Missouri Botanical Garden, St. Louis, USA.

Evans M.T and Faden R.B. 2000. Phylogenetic relationship in Commelinaceae. Systematic Botany 25(4): 668-691.

Faden R.B. 1983. Phytogeography of African Commelinaceae. Bothalia 14: $553-557$.

Faden R.B. 1985. The Families of Monocotyledons. Springer, Berlin, Germany.

Faden R.B. and Hunt D.R. 1991. The classification of Commelinaceae. Taxon 40: 19-31.

Faden R.B. and Inman K.E. 1996. Leaf Anatomy of African Genera of Commelinaceae: Anthericopsis and Murdannia. The Biodiversity of African Plants. Kluwer Academic Publisher, Netherlands.

Hunt D.R. and Poole M.M. 1979. Pollen morphology and the taxonomy of Commelinaceae. Kew Bulletin 33: 406.

Kubitzia K. 1998. The Families and Genera of Vascular Plants. Springer, New York, USA.

Noltie H.J. 2000. Flora of Bhutan. Royal Botanic Garden Edinburgh, Edinburgh, UK.

Press J.R., Shrestha K.K. and Sutton D.A. 2000. Annotated Checklist of the Flowering Plants of Nepal. The Natural History Museum, London, UK.

Tomlinson P.B. 1966. Anatomical data in classification of Commelinaceae. Journal of Linnean Society of Botany 59: 371-395.

Tomlinson P.B. 1969. Anatomy of Monocotyledons. Clarendon Press, Oxford, UK. 